\title{
Solar Sail Lyapunov and Halo Orbits in the Earth-Moon Three-Body Problem
}

\author{
Jeannette Heiligers, ${ }^{a^{*}}$, Sander Hiddink, ${ }^{b \dagger}$ Ron Noomen, ${ }^{\text {b‡ }}$ and Colin R. McInnes ${ }^{a) \S}$ \\ ${ }^{a}$ Advanced Space Concepts Laboratory, Department of Mechanical and Aerospace Engineering, University of \\ Strathclyde, 75 Montrose Street, Glasgow G1 1XJ, Scotland, United Kingdom. \\ ${ }^{b}$ Faculty of Aerospace Engineering, Delft University of Technology, Kluyverweg 1, 2629 HS Delft, the Netherlands
}

Solar sailing has been proposed for a range of novel space applications, including hovering above the ecliptic for high-latitude observations of the Earth and monitoring the Sun from a sub- $\mathrm{L}_{1}$ position for space weather forecasting. These applications, and many others, are all defined in the Sun-Earth threebody problem, while little research has been conducted to investigate the potential of solar sailing in the Earth-Moon three-body problem. This paper therefore aims to find solar sail periodic orbits in the Earth-Moon three-body problem, in particular Lagrange-point orbits. By introducing a solar sail acceleration to the Earth-Moon three-body problem, the system becomes non-autonomous and constraints on the orbital period need to be imposed. In this paper, the problem is solved as a twopoint boundary value problem together with a continuation approach: starting from a natural Lagrange-point orbit, the solar sail acceleration is gradually increased and the result for the previous sail performance is used as an initial guess for a slightly better sail performance. Three in-plane steering laws are considered for the sail, two where the attitude of the sail is fixed in the synodic reference frame (perpendicular to the Earth-Moon line) and one where the sail always faces the Sun. The results of the paper include novel families of solar sail Lyapunov and Halo orbits around the Earth-Moon $\mathrm{L}_{1}$ and $\mathrm{L}_{2}$ Lagrange points, respectively. These orbits are double-revolution orbits that wind around or are off-set with respect to the natural Lagrange-point orbit. Finally, the effect of an out-of-plane solar sail acceleration component and that of the Sun-sail configuration is investigated, giving rise to additional families of solar sail periodic orbits in the Earth-Moon three-body problem.

Keywords: Solar sailing, Circular restricted three-body problem, Earth-Moon problem, Lagrange point orbits, Lyapunov orbits, Halo orbits

\footnotetext{
* Corresponding author. Tel.:+44 141548 5989. E-mail address: jeannette.heiligers@ strath.ac.uk

† E-mail address: s.hiddink@ student.tudelft.nl

$\$$ E-mail address: r.noomen@tudelft.nl

$\S$ E-mail address: colin.mcinnes@strath.ac.uk
} 


\section{Introduction}

Solar sail technology is rapidly gaining momentum after recent successes such as JAXA's IKAROS mission [1] and NASA's NanoSail-D2 mission [2]. Research in the field is flourishing (e.g. through the previously proposed Sunjammer mission [3]) and new solar sail initiatives are scheduled for the future, including The Planetary Society's LightSail-1 mission [4] (launch 2016). Additional proposals include NASA's Lunar Flashlight and NEA Scout missions [5].

The potential of solar sailing lies in the fact that, contrary to other low-thrust propulsion technologies like electric propulsion, it does not rely on an on-board propellant source. By exploiting the radiation pressure generated by solar photons impinging on and reflecting off a large, highly reflective membrane, solar sails can produce a continuous thrust force that is only limited by the lifetime of the membrane material in the space environment. They therefore enable long-lived and high-energy mission concepts that have abundant novel applications [6]. The only real limitations of solar sails are their inability to produce an acceleration component in the direction of the Sun and the fact that the solar radiation pressure decreases with the distance to the Sun squared, limiting their applications in the outer Solar System.

One dynamical system commonly used to describe the motion of the solar sail is the Sun-Earth circular restricted three-body problem (CR3BP). This well-known system yields five natural equilibrium solutions (the $\mathrm{L}_{1}$ to $\mathrm{L}_{5}$ Lagrange points). Adding a propulsive thrust force, such as the one generated by a solar sail, to the CR3BP complements these five Lagrange points with an infinite set of artificial equilibrium points (AEPs). The literature proposes the use of these AEPs to hover along the Sun-Earth line for space weather forecasting [3]. In addition, periodic orbits around these AEPs [7] have been suggested for further space weather monitoring capabilities [8] and parking a spacecraft above the orbit of the Earth for high-latitude observations, navigation and communication [9].

A dynamical system less investigated for solar sailing is the Earth-Moon CR3BP, the most likely reason being that the system is non-autonomous, i.e., the system of differential equations describing the dynamics are explicitly dependent on time. This time dependency is introduced by the Sun's motion around the Earth-Moon system once per synodic lunar period. As a result, the direction of the photons impinging on the solar sail changes, which influences the magnitude and/or direction of the available solar sail acceleration over time. As a result, AEPs in the Earth-Moon problem are no longer stationary: their coordinates are time-dependent and either control is required to compensate for the moving Sun-line or the sail has to navigate along the time-changing AEPs [10]. Finding solar sail periodic orbits in the Earth-Moon system adds another complexity in that the period of the orbit has to be equal to a fraction (or multiple) of the synodic lunar period in order for the orbit to be periodic.

Previous work on solar sail periodic orbits in the Earth-Moon system either linearised the equations of motion [11, 12] or searched for bespoke orbits (e.g. below the lunar South Pole [13]) by solving the optimal control problem. This work will instead look for entire families of solar sail periodic orbits, in particular Lyapunov and Halo orbits, in the Earth-Moon system by solving the accompanying boundary value problem. The existence of such families, closer 
to Earth than possible in the Sun-Earth system, can potentially give rise to unrivalled Earth observation, lunar South Pole coverage and lunar far-side communication capabilities.

The structure of the paper is as follows. First, the non-autonomous dynamical system is described in Section 2, followed by a definition of the two-point boundary value problem (BVP) to be solved in Section 3. Section 4 subsequently describes the initial guess and continuation scheme used to solve the two-point BVP for increasing solar sail performances. Then, after describing the adopted in-plane solar sail steering laws in Section 5, the results are presented in Sections 6 and 0. As the main purpose of this paper is to prove the existence of solar sail periodic Lagrange-point orbits, the results of two particular cases will be provided: solar sail Lyapunov orbits at the $\mathrm{L}_{1}$-point and solar sail Halo orbits at the $\mathrm{L}_{2}$-point. Finally, in Section 8 the potential of an out-of-plane solar sail acceleration component is investigated, while the effect of the Sun-sail configuration at time $t=0$ is investigated in Section 9. The paper ends with the conclusions.

\section{Dynamical system}

In the well-known circular restricted three-body problem (CR3BP), the motion of an object with an infinitely small mass $m$ (i.e. the solar sail spacecraft) is described under the influence of the gravitational attraction of two much larger primary objects with masses $m_{1}$ (the Earth) and $m_{2}$ (the Moon). The gravitational influence of the small object on the primaries is neglected and the primaries are assumed to move in circular (co-planar) orbits about their common centre-of-mass.

Figure 1 shows the reference frame that is employed in the CR3BP: the origin coincides with the centre-of-mass of the system, the $x$-axis connects the primaries and points in the direction of the smaller of the two, $m_{2}$, while the $z$ axis is directed perpendicular to the plane in which the two primaries move. The $y$-axis completes the right-handed reference frame. Finally, the frame rotates at constant angular velocity $\omega$ about the $z$-axis: $\boldsymbol{\omega}=\omega \hat{\mathbf{z}}$.

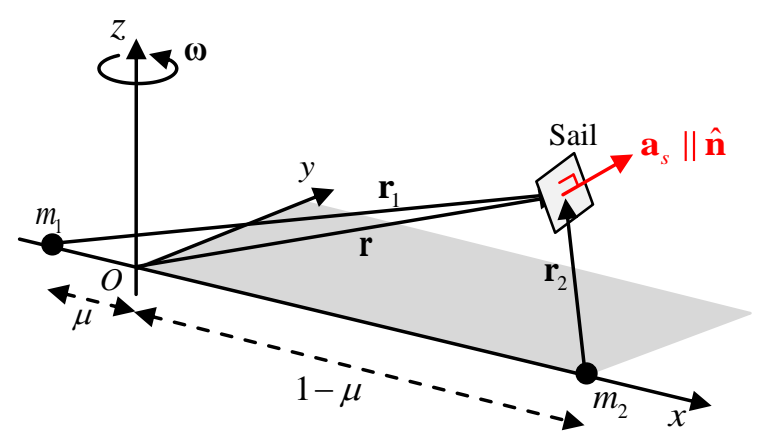

Figure 1 Schematic of circular restricted three-body problem 
Table 1 Earth-Moon CR3BP parameters

\begin{tabular}{lc}
\hline & Earth-Moon CR3BP \\
\hline$\mu$ & 0.01215 \\
Total mass $\left(m_{1}+m_{2}\right)$ & $6.04718 \times 10^{24} \mathrm{~kg}$ \\
Earth-Moon distance & $384,401 \mathrm{~km}$ \\
One time unit & $3.7749 \times 10^{5} \mathrm{~s}$ \\
$\Omega_{S}$ & 0.9252 \\
\hline
\end{tabular}

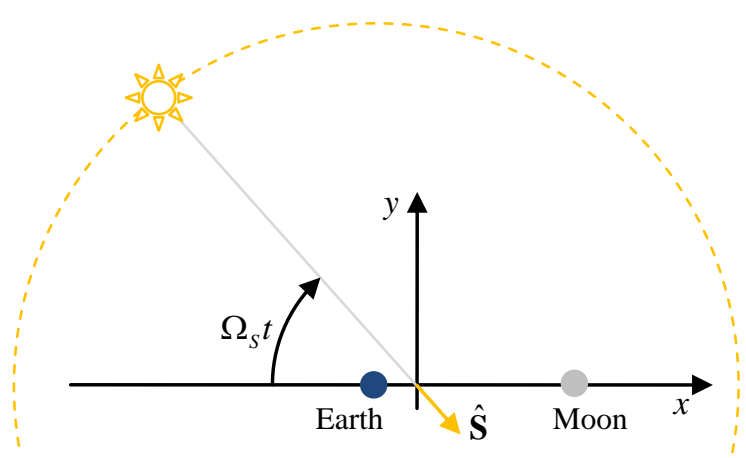

Figure 2 Schematic of non-autonomous Earth-Moon three-body problem

In this reference system, the motion of the solar sail is described by: [6]

$$
\ddot{\mathbf{r}}+2 \boldsymbol{\omega} \times \dot{\mathbf{r}}+\boldsymbol{\omega} \times(\boldsymbol{\omega} \times \mathbf{r})=\mathbf{a}_{s}-\nabla V
$$

with $\mathbf{r}=\left[\begin{array}{lll}x & y & z\end{array}\right]^{T}$ the position vector of the sail. The terms on the left-hand side are the kinematic, coriolis and centripetal accelerations, respectively, while the terms on the right-hand side are the solar sail acceleration and the gravitational acceleration exerted by the primary masses. The gravitational potential $V$ is given by:

$$
V=-\left(\frac{1-\mu}{r_{1}}+\frac{\mu}{r_{2}}\right)
$$

with the vectors $\mathbf{r}_{1}$ and $\mathbf{r}_{2}$ defined as $\mathbf{r}_{1}=\left[\begin{array}{lll}x+\mu & y & z\end{array}\right]^{T}$ and $\mathbf{r}_{2}=\left[\begin{array}{lll}x-(1-\mu) & y & z\end{array}\right]^{T}$. Following [6], the centripetal acceleration in Eq. (1) can be written as the gradient of a scalar potential function, $\Phi=-\frac{1}{2}\|\boldsymbol{\omega} \times \mathbf{r}\|^{2}$, and can be combined with the gravitational potential into a new, effective potential, $U$ :

$$
U=-\frac{x^{2}+y^{2}}{2}-\left(\frac{1-\mu}{r_{1}}+\frac{\mu}{r_{2}}\right)
$$

The new set of equations of motion then becomes: 


$$
\ddot{\mathbf{r}}+2 \boldsymbol{\omega} \times \dot{\mathbf{r}}=\mathbf{a}_{s}-\nabla U
$$

Finally, for the solar sail acceleration, an ideal sail model is assumed [6]. An ideal solar sail is a sail that is perfectly reflecting and perfectly flat. The incoming solar photons are therefore specularly reflected and the solar radiation pressure force acts perpendicular to the sail surface, in direction $\hat{\mathbf{n}}$, see Figure 1. Furthermore assuming that the solar radiation pressure is constant in magnitude throughout the Earth-Moon system, the solar sail acceleration $\mathbf{a}_{s}$ can be written as:

$$
\mathbf{a}_{s}=a_{0, E M}(\hat{\mathbf{S}}(t) \cdot \hat{\mathbf{n}})^{2} \hat{\mathbf{n}}
$$

In Eq. (5), $\hat{\mathbf{S}}$ is the direction of the Sun-line, see Figure 2, which can be expressed as:

$$
\hat{\mathbf{S}}=\left[\begin{array}{lll}
\cos \left(\Omega_{S} t\right) & -\sin \left(\Omega_{S} t\right) & 0
\end{array}\right]^{T}
$$

with $\Omega_{S}$ the angular rate of the Sun-line in non-dimensional units, see also Table 1. Note that Eq. (6) ignores the small inclination difference between the Sun-Earth and Earth-Moon orbital planes and at time $t=0$, the Sun is assumed to be on the negative $x$-axis.

Finally, note that $a_{0, E M}$ is the sail's characteristic acceleration in non-dimensional units. The characteristic acceleration is the acceleration generated by the solar sail when it faces the Sun at Earth's distance (at 1 Astronomical Unit). Derived from the Sunjammer sail performance, a typical value for this characteristic acceleration is $0.215 \mathrm{~mm} / \mathrm{s}^{2}$ [14], which translates into a value for $a_{0, E M}$ of 0.0798 .

\section{Boundary value problem}

Due to the non-autonomous behaviour of the system, periodic orbits are found by treating the problem as a two-point boundary value problem (BVP) rather than using conventional methods such as differential correctors [15]. The dynamics of the BVP are given in Eq. (4) and the boundary constraints are given by:

$$
\mathbf{x}\left(t_{0}\right)=\mathbf{x}\left(t_{f}\right)
$$

with $\mathbf{x}=\left[\begin{array}{cc}\mathbf{r} & \dot{\mathbf{r}}\end{array}\right]^{T}$, the indices ' 0 ' and ' $f$ ' indicating initial and final conditions and $t_{f}=2 \pi / \Omega_{S}$, i.e. one synodic lunar period. Please note that $\Omega_{S} \neq \omega$ due to the angular distance travelled by the Earth-Moon system around the Sun in one sidereal lunar period.

The BVP is solved using the collocation method implemented in the MATLAB ${ }^{\circledR} b v p 4 c . m$ function. 


\section{$4 \quad$ Initial guess}

To solve the two-point boundary value problem, bvp4c.m needs an initial guess. Here, a continuation approach is adopted, where the search for solar sail periodic orbits starts from a natural periodic orbit with a suitable period. The sail performance in terms of characteristic acceleration $a_{0, E M}$ is then slowly increased and the solution for the previous value for $a_{0, E M}$ is used as initial guess for the next value for $a_{0, E M}$. This will give rise to families of periodic orbits for increasing value of the sail performance.

\subsection{Lyapunov orbit around L1}

The selection of the initial, natural Lyapunov orbit around the $\mathrm{L}_{1}$ Lagrange point is shown in Figure 3a. These natural Lyapunov orbits are generated using the approach described in Reference [15] and their orbital periods are provided in Figure 3b. Only the Lyapunov orbits with a period equal to a fraction of the synodic lunar period would be suitable initial guess candidates, i.e. the following constraint applies:

$$
P_{\text {Nat Lyap }}=\frac{1}{i} \frac{2 \pi}{\Omega_{S}} \text { with } i=1,2,3 \ldots
$$

The orbit that fulfils this constraint is indicated with a thick blue line in plot a) and with a blue asterisk in plot b). The selected Lyapunov orbit has a period of $P_{\text {Nat Lyap }}=\frac{1}{2} \frac{2 \pi}{\Omega_{S}}$ and thus makes two orbital revolutions in one synodic lunar period.

a)

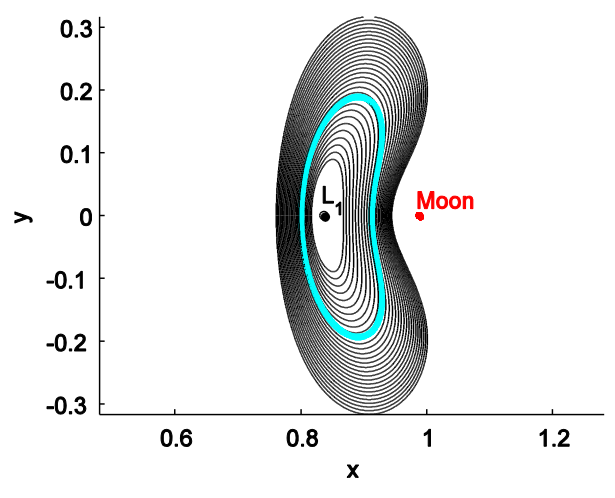

b)

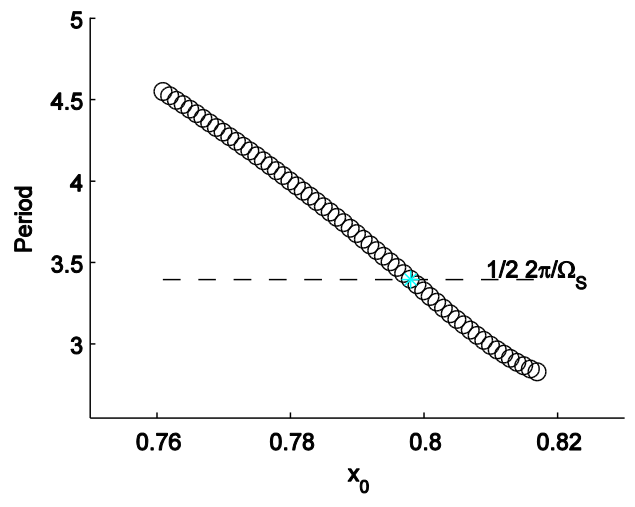

Figure 3 Lyapunov family at $L_{1}$. a) Lyapunov orbits. b) Period of Lyapunov orbits in a).

\subsection{Halo orbit around $\mathrm{L}_{2}$}

The approach to find the initial, natural Halo orbit around the $\mathrm{L}_{2}$ Lagrange point is very similar to the approach described for the Lyapunov orbit around $\mathrm{L}_{1}$. The family of natural (northern) Halo orbits around $\mathrm{L}_{2}$ is provided in Figure $4 \mathrm{a}$ with their orbital periods in Figure $4 \mathrm{~b}$. Again, the orbit with a period equal to a fraction of the synodic 
lunar period is chosen as suitable initial guess, which in this case also has a period of $P_{\text {Nat Halo }}=\frac{1}{2} \frac{2 \pi}{\Omega_{S}}$ and therefore makes two orbital revolutions in one synodic lunar period.

a)

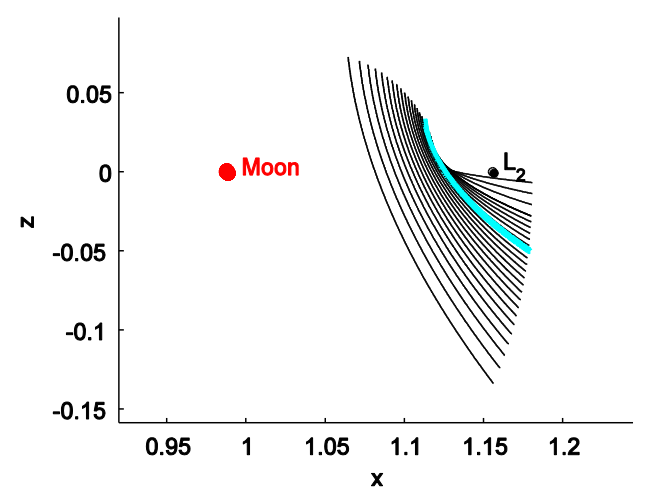

b)

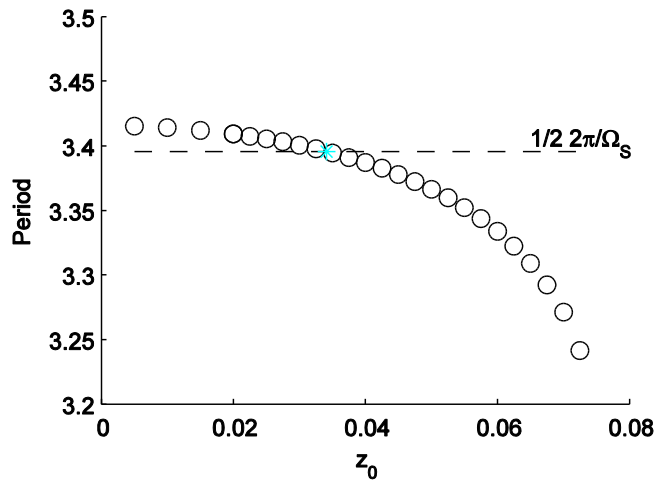

Figure 4 Northern Halo family at $L_{2}$. a) Halo orbits. b) Period of Halo orbits in a).

\section{$5 \quad$ Solar sail steering laws}

Different families of solar sail periodic orbits can be generated for different solar sail steering laws. The three laws investigated in this paper are:

1) The sail normal is always directed along the Earth-Moon line, i.e. along the $x$-axis:

$$
\hat{\mathbf{n}}=\operatorname{sign}\left(\cos \left(\Omega_{S} t\right)\right)\left[\begin{array}{lll}
1 & 0 & 0
\end{array}\right]^{T}
$$

This steering law allows for a constant attitude of the sail in the CR3BP reference frame (and therefore a constant acceleration direction), but implies a changing solar sail acceleration magnitude. Note that the term $\operatorname{sign}\left(\cos \left(\Omega_{S} t\right)\right)$ takes into account that the solar sail normal vector changes sign when the Sun moves from a position where it illuminates the Earth-facing side of the sail to a position where it illuminates the Moon-facing side of the sail. Note that this implies that the sail would have to be reflective on both sides. However, conventionally, solar sails are only reflective on one side, the front side (e.g. the Earth-facing side), and will have a highly thermally emitting rear surface. This is to emit the absorbed energy of the small fraction of incident solar radiation that, in reality, will be absorbed by the sail substrate [6]. The rear surface should therefore not be exposed to sunlight. For the steering law in Eq. (9) this would require the solar sail to be rotated over $180 \mathrm{deg}$ once per synodic lunar period, which introduces a severe steering effort. Therefore, an alternative, second control law is adopted as described below.

2) The sail normal is always directed along the Earth-Moon line, i.e. along the $x$-axis, but the sail is 'switched off' when the Sun illuminates the rear side of the sail: 


$$
\begin{array}{ll}
\hat{\mathbf{n}}=\left[\begin{array}{lll}
1 & 0 & 0
\end{array}\right]^{T} & \text { if }(\hat{\mathbf{S}} \cdot \hat{\mathbf{n}}) \geq 0 \\
\mathbf{a}_{s}=\mathbf{0} & \text { if }(\hat{\mathbf{S}} \cdot \hat{\mathbf{n}})<0
\end{array}
$$

Note that a zero acceleration can be achieved by positioning the sail edge-wise to the Sun-line. Also note that $(\hat{\mathbf{S}} \cdot \hat{\mathbf{n}})=\cos \left(\Omega_{s} t\right)$ and therefore the constraint $\mathbf{a}_{s}=\mathbf{0}$ is imposed when $\frac{1}{2} \pi \leq t \leq \frac{3}{2} \pi$. This control law will be referred to as the constrained Earth-Moon line steering law.

3) The sail normal is always directed along the Sun-sail line, i.e. the sail always faces the Sun:

$$
\hat{\mathbf{n}}=\hat{\mathbf{S}}
$$

Contrary to the first and second steering laws, this steering law allows for a constant magnitude of the sail acceleration ( $\mathbf{a}_{s}=a_{0, E M} \hat{\mathbf{S}}$ ), but implies a changing attitude of the sail in the CR3BP reference frame. However, the sail's attitude with respect to the Sun-sail line remains fixed and this Sun-pointing orientation can be achieved passively through an offset between the centre of mass and centre of pressure [16].

\section{Results - $\mathrm{L}_{1}$ Lyapunov orbits}

This section presents the families of solar sail Lyapunov orbits around the Earth-Moon $\mathrm{L}_{1}$-point and for the three steering laws outlined in the previous section. The maximum solar sail characteristic acceleration considered is $a_{0, E M}=0.088$, which is slightly larger than what was proposed for the Sunjammer mission, but indicates what could be feasible in the near-term.

The results are summarised in Figure 5, which shows both the families of orbits as well as particular orbits for $a_{0, E M}=0.088$. The families originate from the natural Lyapunov orbit (see Figure 3) and are created by slowly increasing the characteristic acceleration, $a_{0, E M}$, of the solar sail. The larger the value for $a_{0, E M}$, the more the solar sail orbit deviates from the natural Lyapunov orbit. Note that the asterisks in plots $b, d$ and $f$ indicate the initial condition of the orbit, which is chosen to be the $y$-axis crossing on the Earth-side of the $\mathrm{L}_{1}$-point. Section 9 will investigate the influence of choosing a different initial condition on the shape of the solar sail periodic orbits.

Figure 5 clearly shows the effect of adding a solar sail acceleration to the natural Lyapunov orbits, the doublerevolution motion as well as the effect of the different steering laws. In general, the more the sail is exploited, the larger the offset between the natural and solar sail Lyapunov orbits becomes: the constrained Earth-Moon line steering law (plots c-d) that only uses the sail during half of the orbital period and only exploits the maximum solar sail acceleration at time $t=0$ shows the smallest offset. This offset is created during the first half of the first revolution and the second half of the second revolution and shows a displacement in the direction opposite to the direction to the sail normal $\hat{\mathbf{n}}$ (i.e. the sail acceleration vector). 
Instead, the non-constrained Earth-Moon line steering law (plots a-b) shows a displacement throughout the orbital period, towards the left when the Sun illuminates the Earth-facing side of sail and towards the right when the Sun illuminates the Moon-facing side of the sail. However, the greatest offset between the natural and solar sail Lyapunov orbits is achieved for the Sun-sail line steering law as the maximum sail acceleration is exploited throughout the entire orbital period. The result is a set of solar sail Lyapunov orbits with an inner and outer revolution that are smaller and greater than the natural Lyapunov orbit, respectively.

\section{$7 \quad$ Results $-\mathbf{L}_{2}$ Halo orbits}

This section presents the families of solar sail Halo orbits around the $\mathrm{L}_{2}$-point of the Earth-Moon system and for the three steering laws as outlined in Section 5. The results are summarised in Figure 6 (family of orbits) and Figure 7 (for particular solar sail characteristic accelerations).

When considering the in-plane motion of the three families (in Figure 6a, c and e) very similar conclusions can be drawn as for the Lyapunov orbits in Section 6 and Figure 5 (keeping in mind that the initial condition for the Halo orbits is the $y$-axis crossing on the far-side of the $\mathrm{L}_{2}$-point): for the constrained Earth-Moon line steering law (plots $\mathrm{c}, \mathrm{d}$ ), the portion of the orbit where the solar sail acceleration acts (i.e. the right side of the orbit) is displaced in the direction opposite to the sail normal vector. Due to the flip in the direction of the normal vector for the non-constrained EarthMoon line steering law (plots a, b), the orbit is displaced in both directions with respect to the natural Halo orbit. Finally, the Sun-sail line steering law (plots e, f) provides the largest off-set with respect to the natural orbit and creates an in-plane inner and outer loop.

Most interesting is the out-of-plane motion of the solar sail Halo orbits. The effect of the solar sail acceleration is clear from plots b, $\mathrm{d}$ and $\mathrm{f}$ : the better the performance of the sail, the more the Halo orbit is flattened, positioning the southern part of the orbit closer to the Moon. For a large enough sail characteristic acceleration, the Halo orbit even reduces to a planar Lyapunov orbit for the constrained Earth-Moon and Sun-sail line steering laws. More details on the out-of-plane motion can be found in Figure 7 which shows the solar sail Halo orbits for $a_{0, E M}=0.088$. Note that an additional result for the Sun-sail line steering law and $a_{0, E M}=0.044$ is added to show the progression of the out-of-plane motion when the sail performance is increased. 
Earth-Moon line steering law

a)

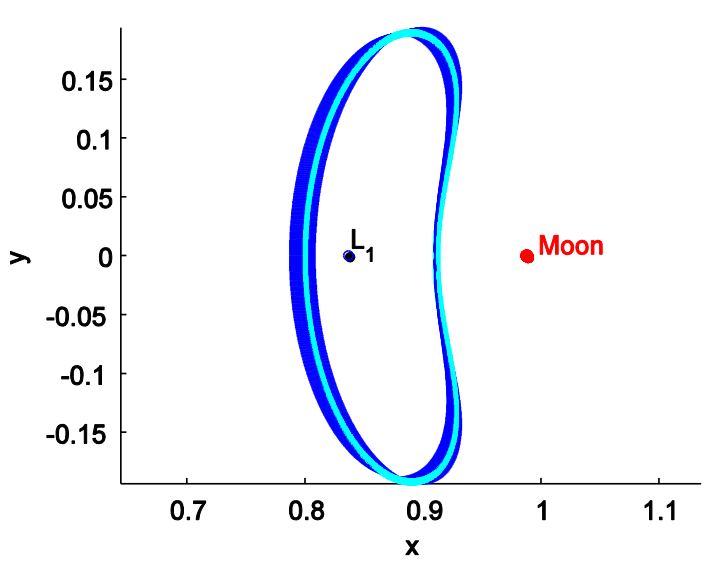

b)

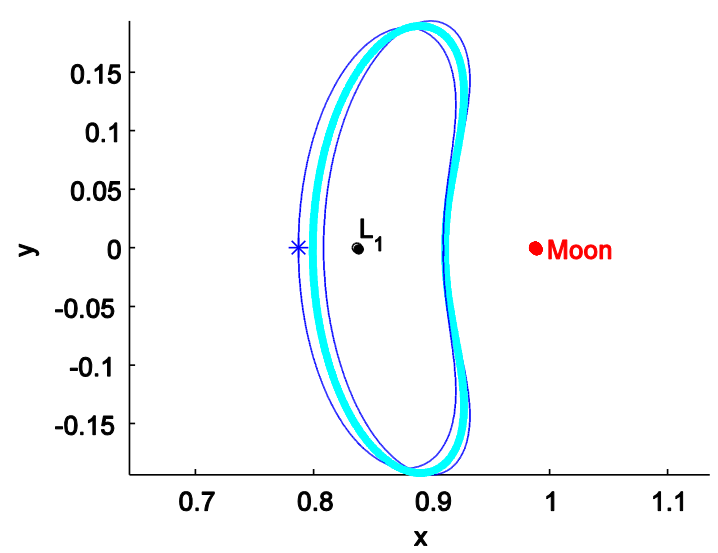

Constrained Earth-Moon line steering law

c)

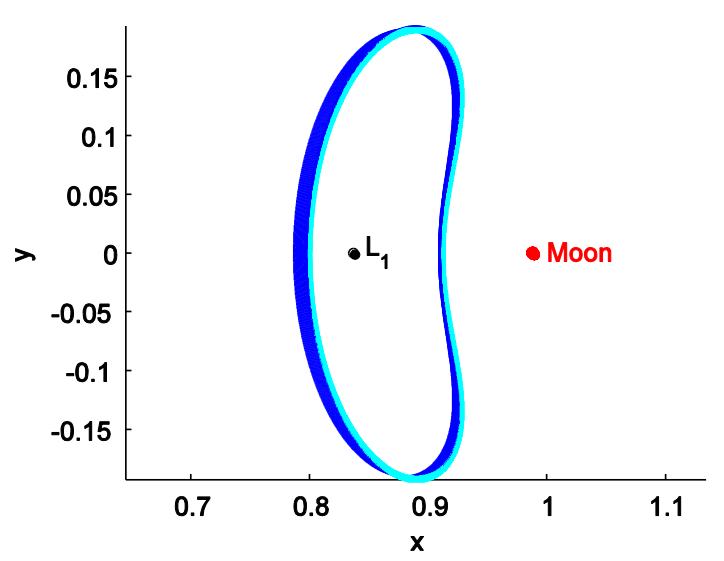

d)

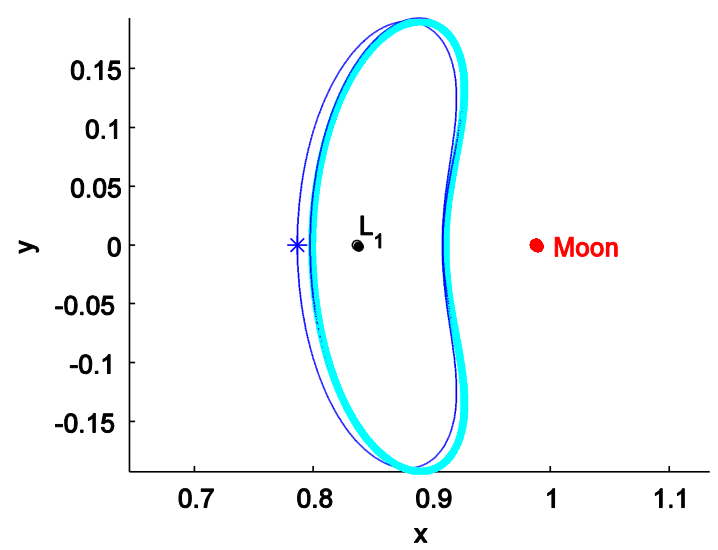

Sun-sail line steering law

e)

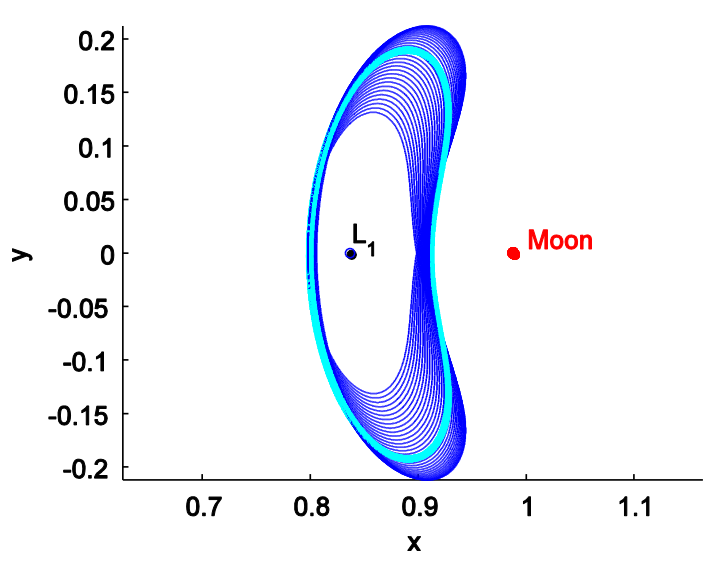

f)

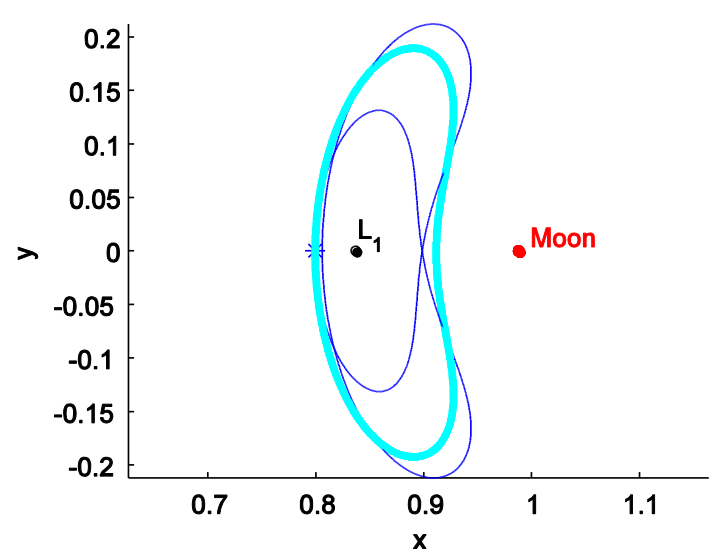

Figure 5 Solar sail Lyapunov orbits at $L_{1}$ with different solar sail steering laws. The thick cyan orbit is the natural Halo orbit in Figure 3a. a, c, e) Family of orbits for various values for $a_{0, E M}$. b, d, e) Orbit for $a_{0, E M}=\mathbf{0 . 0 8 8}$ (the asterisk is the initial condition). 
a)

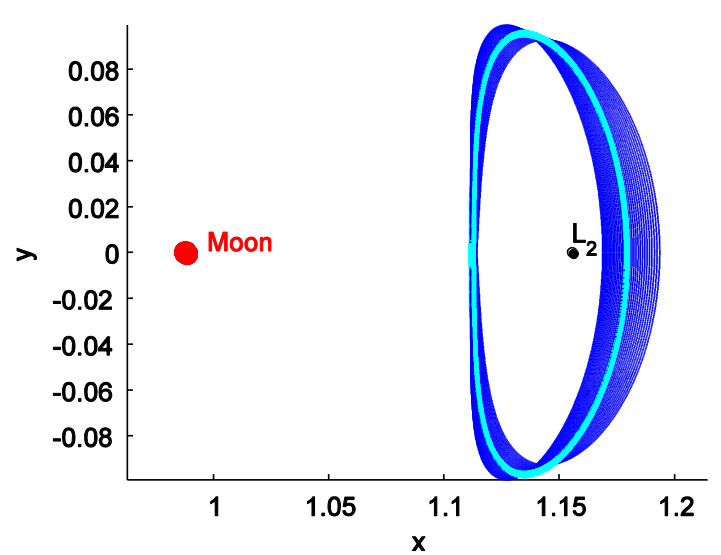

b)

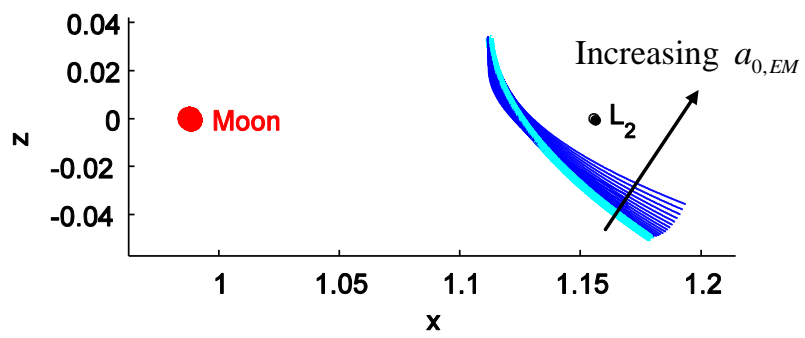

Constrained Earth-Moon line steering law

c)

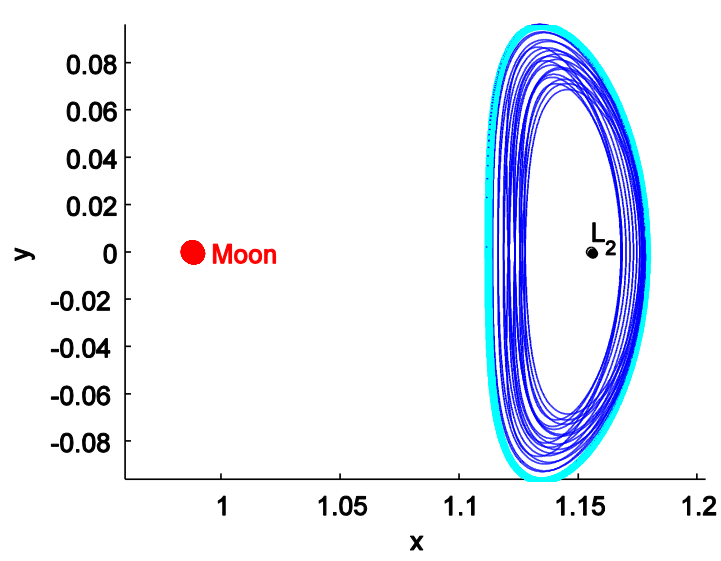

d)

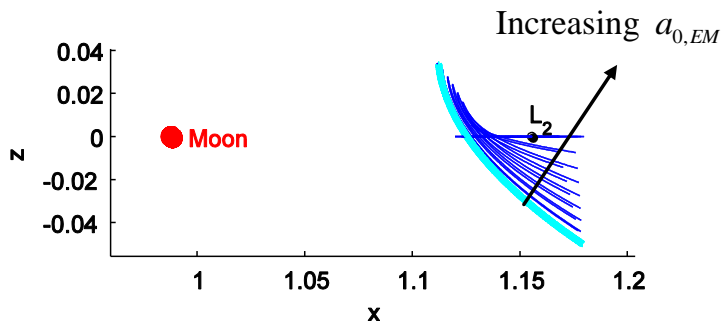

Sun-sail line steering law

e)

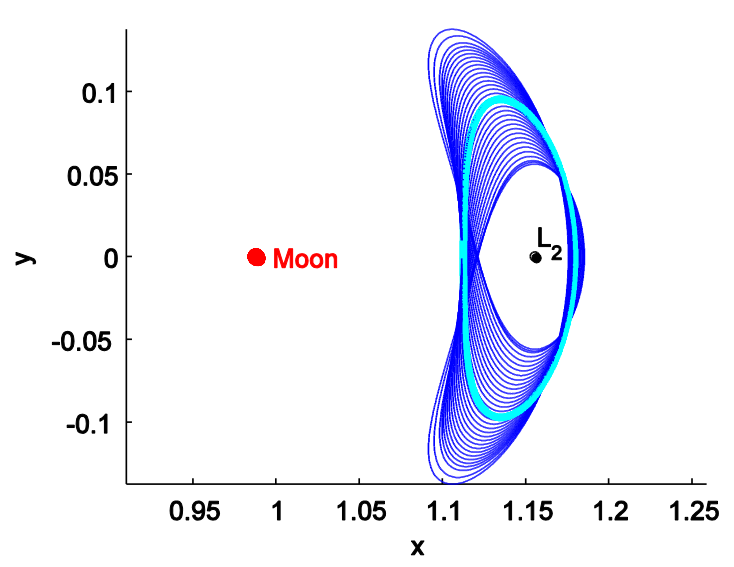

f)

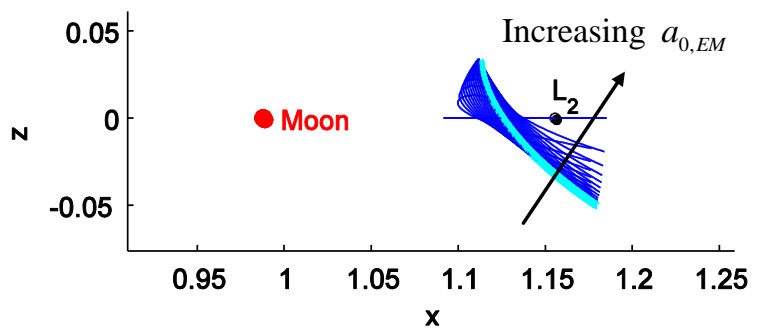

Figure 6 Families of solar sail Halo orbits at $L_{2}$ with different solar sail steering laws and for various values for $a_{0, E M}$. The thick cyan orbit is the natural Halo orbit in Figure 4a. a, c, e) Projections onto the (x,y)plane. b, d, e) Projections onto the $(x, z)$-plane. 
Earth-Moon line steering law

a)

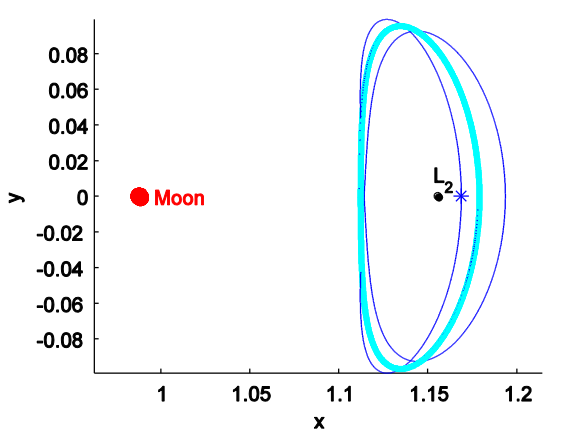

b)

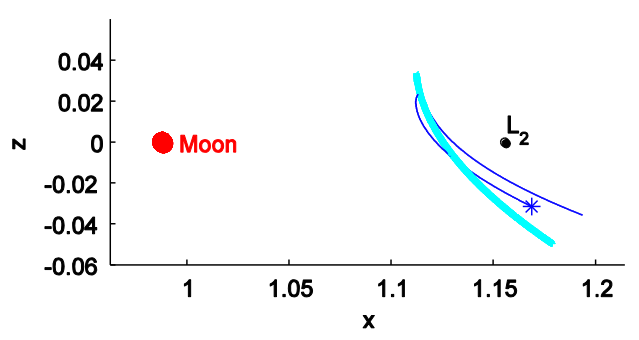

Constrained Earth-Moon line steering law

c)

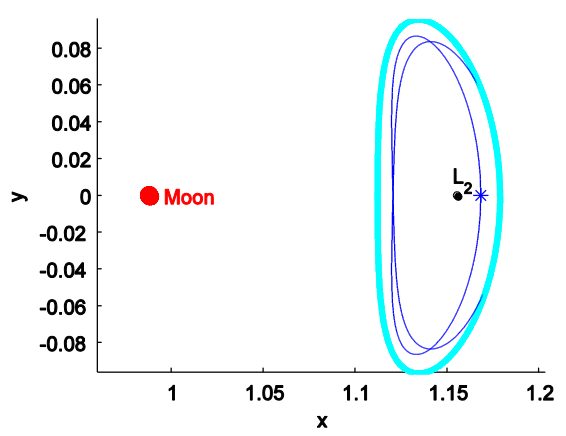

d)

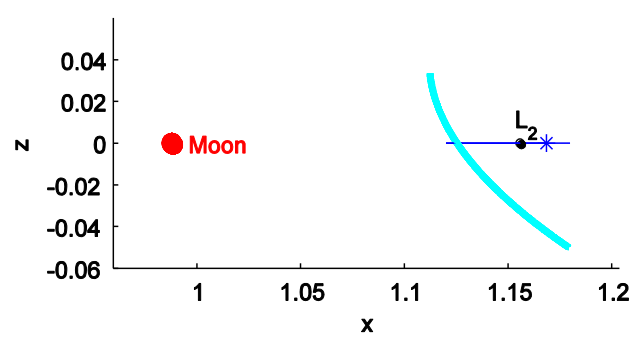

Sun-sail line steering law

e)

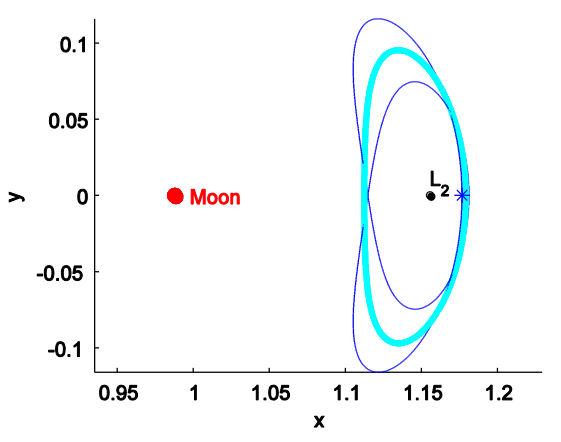

g)

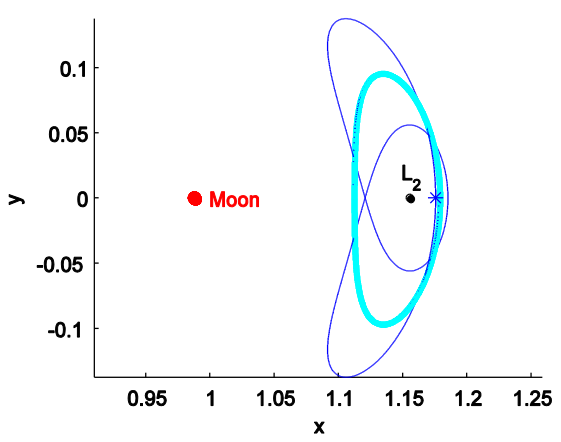

f)

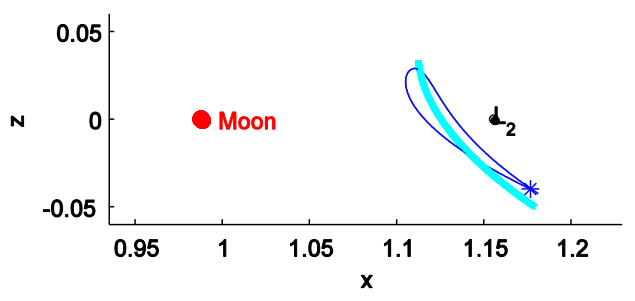

h)

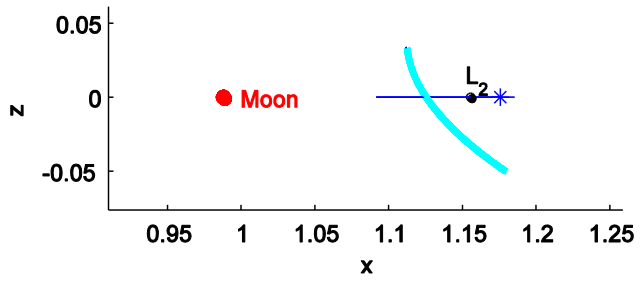

Figure 7 Solar sail Halo orbits at $L_{2}$ with different solar sail steering laws. The thick cyan orbit is the natural

Halo orbit in Figure 4a. The asterisk is the initial condition. a-d, g, h) $a_{0, E M}=\mathbf{0 . 0 8 8}$. e, f) $a_{0, E M}=\mathbf{0 . 0 4 4}$. 


\section{Out-of-plane solar sail acceleration}

The steering laws proposed in Section 5 only consider an in-plane solar sail acceleration. However, the solar sail can be pitched with respect to the Earth-Moon plane to also create an out-of-plane acceleration component. This section will demonstrate the potential of this out-of-plane steering law by using the solar sail Lyapunov orbits at the $\mathrm{L}_{1}$-point with a Sun-sail line steering law as a test case. Then, rather than adopting the in-plane law, $\hat{\mathbf{n}}=\hat{\mathbf{S}}$, the following outof-plane Sun-sail line steering law is considered, see also Figure 8:

$$
\hat{\mathbf{n}}=\left[\begin{array}{lll}
\cos \gamma \cos \left(\Omega_{S} t\right) & -\cos \gamma \sin \left(\Omega_{S} t\right) & \sin \gamma
\end{array}\right]^{T}
$$

Note that for a pitch angle $\gamma=0$, Eq. (12) reduces to the in-plane steering law.

To generate the family of out-of-plane Sun-sail line Lyapunov orbits at $\mathrm{L}_{1}$, a continuation in $\gamma$ is applied: starting from the planar solar sail Lyapunov orbit with $\gamma=0$ and $a_{0, E M}=0.088$ in Figure 5f, the value for the pitch angle is slowly increased, the BVP is solved and the result is used as an initial guess for a slightly larger value for $\gamma$. The family of orbits that results from this is provided in Figure $9 a-b$ for a range of pitch angles, $\gamma=[-60,60]$ in deg. Note that the orbits for negative and positive values for the pitch angles are equal, only mirrored in the $(x, y)$-plane, and create out-of-plane displacements above and below the $(x, y)$-plane, respectively. The maximum out-of-plane displacement that is achieved in each orbit of the family is provided in Figure 10, which clearly shows that for a pitch angle of $\gamma= \pm 34.5 \mathrm{deg}$, the orbit extends farthest above or below the Earth-Moon plane. The corresponding orbit is depicted in Figure 9c and reaches an out-of-plane displacement of approximately 10,250 km.

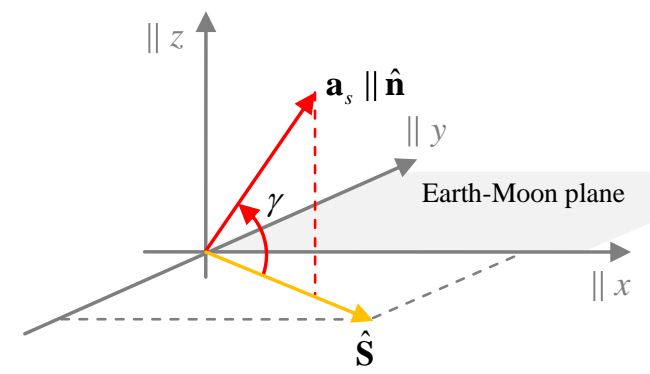

Figure 8 Schematic of out-of-plane Sun-sail line steering law 
a)

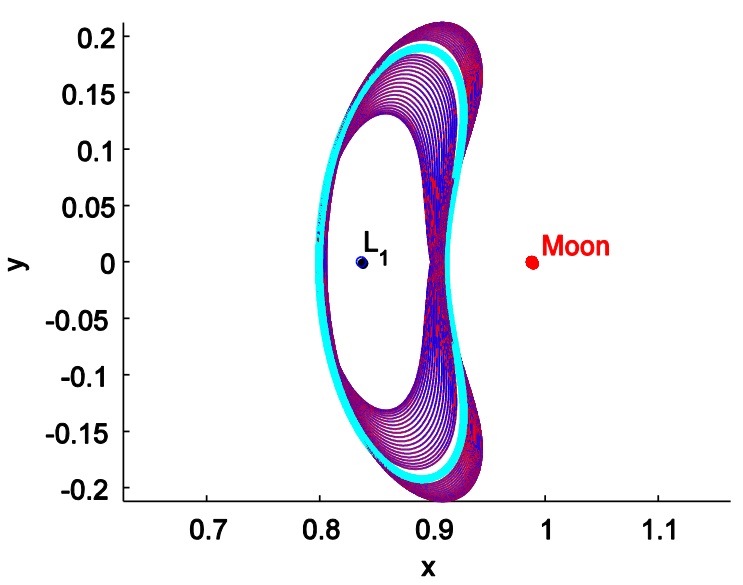

b)

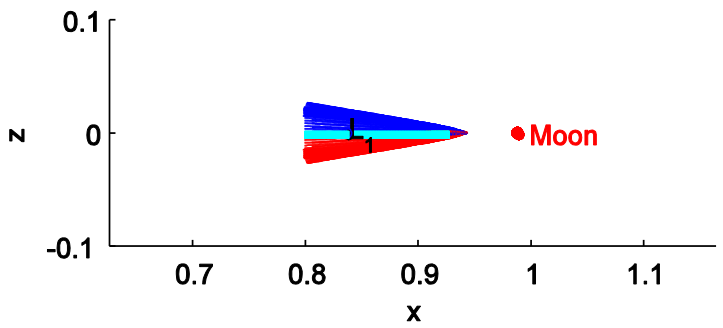

c)

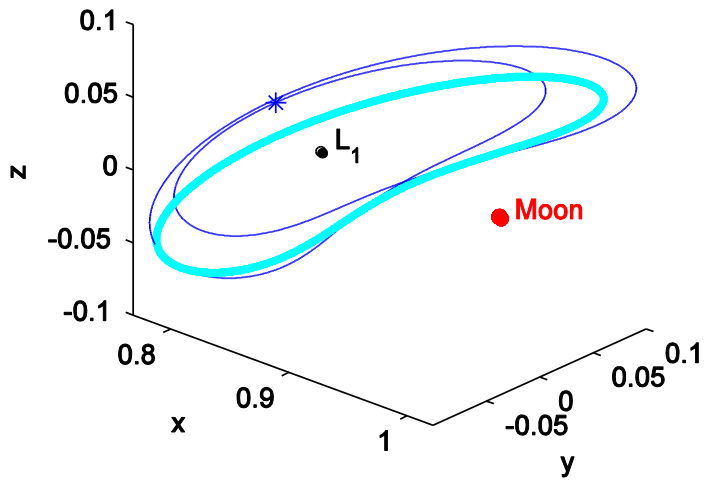

Figure 9 Solar sail Lyapunov orbits at $\mathbf{L}_{1}$ with out-of-plane Sun-sail line steering law and $a_{0, E M}=0.088$.

Orbits in blue and red are for $\gamma>0$ and $\gamma<0$, respectively. The thick cyan orbit is the natural Halo orbit. a-b) Family of orbits projected on the $(x, y)$ - and $(x, z)$-planes. c) Orbit with maximum out-of-plane displacement ( $\gamma=34.5 \mathrm{deg}$ ) (the asterisk is the initial condition). 


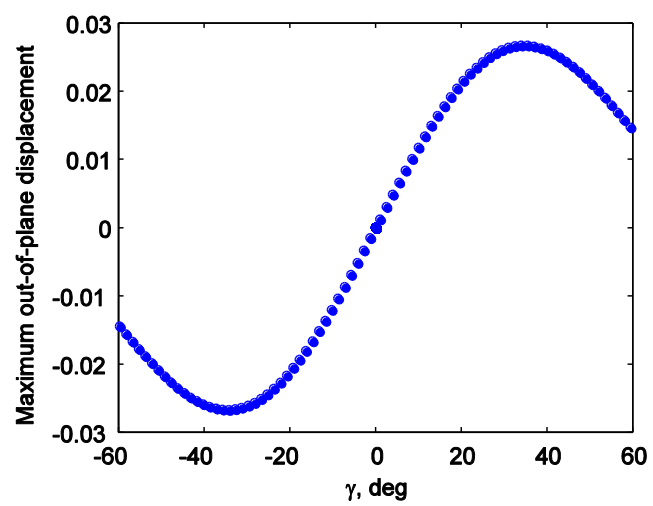

Figure 10 Maximum out-of-plane displacement in each orbit of the family of solar sail Lyapunov orbits at $\mathrm{L}_{1}$ with out-of-plane Sun-sail line steering law (Figure 9) as a function of the solar sail pitch angle, $\gamma$.

\section{Initial condition at time $\mathbf{t}=\mathbf{0}$}

As indicated in Section 2, the position of the Sun at time $t=0$ is assumed to be on the negative $x$-axis. The actual Sun-sail configuration throughout the orbit and during one synodic lunar period then depends on the choice for the initial condition along the natural Halo orbit from where the continuation for solar sail periodic orbits starts. The choice for this initial condition will influence the shape of the families of solar sail periodic orbits, which is demonstrated in this section by investigating an alternative initial condition for the solar sail Halo orbits with a constrained Earth-Moon line steering law, see Figure $6 \mathrm{c}$ and d. As mentioned before, the results of Section 0 are obtained by choosing the initial condition to be the $y$-axis crossing on the far-side of the $\mathrm{L}_{2}$-point, i.e. below the Earth-Moon plane. As alternative, this section considers the $y$-axis crossing on the Moon-side of the $\mathrm{L}_{2}$-point, i.e. above the Earth-Moon plane. When doing so, the result in Figure 11 is obtained.

Comparing Figure $6 \mathrm{c}$ and d and Figure 11 immediately shows the effect of the choice for the initial condition: when choosing the Moon-side $y$-axis crossing as initial condition (as is done in Figure 11), the left part of the orbit (where the solar sail acceleration acts at the start of the first revolution and at the end of the second revolution) is displaced in the direction opposite to the sail normal vector, causing the solar sail Halo orbit to increase with respect to the natural Halo orbit. Instead, when choosing the far-side $y$-axis crossing as initial condition (as was done in Figure 6c and d), the right part of the orbit is displaced in the direction opposite to the sail normal vector, causing the solar sail Halo orbit to decrease with respect to the natural Halo orbit. This example demonstrates that strict insertion constraints need to be imposed in order for the solar sail to enter the desired orbit.

Note that the effect on the out-of-plane motion is very similar for both initial conditions, i.e. for large enough solar sail characteristic accelerations the Halo orbit reduces to a planar Lyapunov orbit. 
a)

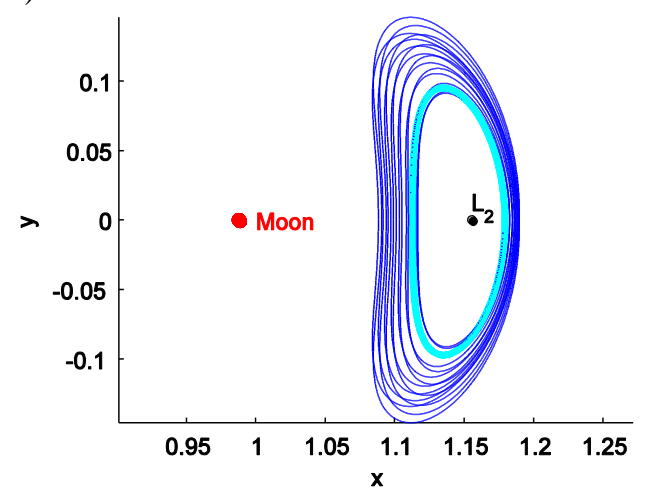

b)

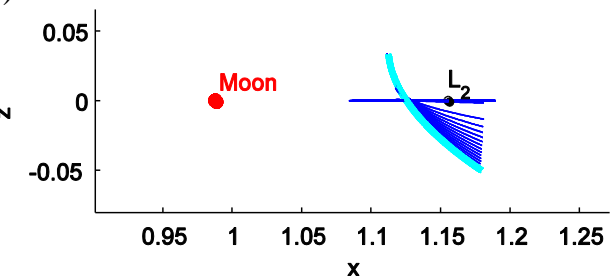

c)

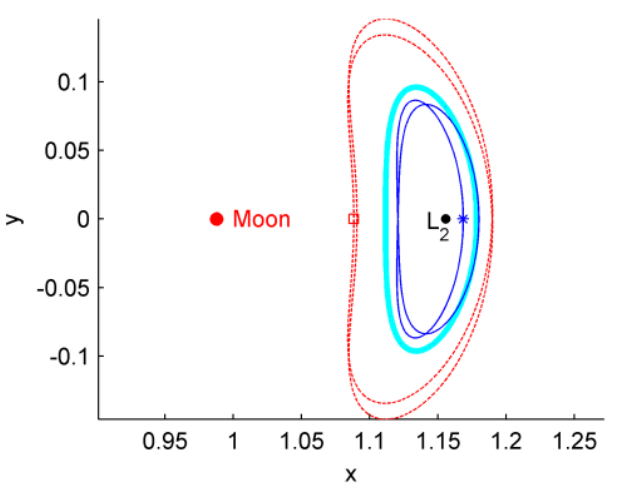

Figure 11 Effect of choice for initial condition at time $t=0$ on the solar sail Halo family at $\mathrm{L}_{2}$ for a constrained Earth-Moon line steering law and for $a_{0, E M}=0.088$. a, b) Choosing the $y$-axis crossing on the Moon-side of $L_{2}$ as initial condition. c) The blue solid line is the solar sail Halo orbit for $r_{0}$ coinciding with the $y$-axis crossing on the far-side of the $\mathrm{L}_{2}$-point (blue asterisk), the red dashed line is the solar sail Halo orbit for $\boldsymbol{r}_{0}$ coinciding with the $y$-axis crossing on the Moon-side of the $L_{2}$-point.

\section{Conclusions}

This paper has demonstrated the existence of solar sail periodic orbits in the non-autonomous Earth-Moon system. By solving the accompanying two-point boundary value problem and using a continuation approach, families of solar sail $\mathrm{L}_{1}$-Lyapunov and $\mathrm{L}_{2}$-Halo orbits have been found for increasing solar sail performances. Due to the nonautonomous behaviour of the system, all orbits have a period of one synodic lunar period and make two revolutions per orbital period. In addition, different families have been obtained for different in-plane solar sail steering laws: either keeping the solar sail perpendicular to the Earth-Moon line or to the Sun-sail line. As the latter can continuously exploit the maximum achievable solar sail acceleration, the families with a Sun-sail line steering law show a greater difference with respect to the natural Lyapunov or Halo orbits from which they bifurcate than the Earth-Moon line steering law. For each law, the in-plane motion of the solar sail periodic orbits winds around the natural Lyapunov/Halo orbit, while for the Halo orbits the solar sail also causes a flattening of the out-of-plane 
motion towards the Earth/Moon plane. For large enough solar sail characteristic accelerations, the Halo orbits even reduce to planar Lyapunov orbits. Furthermore, by introducing an out-of-plane steering law, the family of solar sail Lyapunov orbits at $\mathrm{L}_{1}$ could be extended in the out-of-plane direction, achieving the maximum displacement for a sail pitch angle of $34.5 \mathrm{deg}$. Finally, the effect of different initial conditions, i.e. different initial Sun-sail configurations, has been investigated, showing a significant effect on the solar sail Halo orbits at $\mathrm{L}_{2}$. This implies that insertion conditions are strict in order to ensure that the desired solar sail Halo orbit is achieved.

\section{Acknowledgements}

This work was funded by the European Research Council Advanced Investigator Grant-227571 - Visionary Space Systems: Orbital Dynamics at Extremes of Spacecraft Length-Scale.

\section{References}

1. Tsuda, Y., Mori, O., Funase, R., Sawada, H., Yamamoto, T., Saiki, T., Endo, T., Yonekura, K., Hoshino, H., and Kawahuchi, J. "Achievement of IKAROS - Japanese deep space solar sail demonstration mission," Acta Astronautica Vol. 82, 2013, pp. 183-188. doi: 10.1016/j.actaastro.2012.03.032

2. Johnson, L., Whorton, M., Heaton, A., Pinson, R., Laue, G., and Adams, C. "NanoSail-D: A Solar Sail Demonstration Mission," Acta Astronautica Vol. 68, 2011, pp. 571-575. doi: 10.1016/j.actaastro.2010.02.008

3. Braafladt, A. C., Artusio-Glimpse, A. B., and Heaton, A. F. "Validation of Solar Sail Simulations for the NASA Solar Sail Demonstration Project," AIAA SPACE 2014 Conference and Exposition. San Diego, CA, 2014.

4. Biddy, C., and Svitek, T. "LightSail-1 Solar Sail Design and Qualification," Proceedings of the 41st Aerospace Mechanisms Symposium. Pasadena, CA, 2012.

5. McNutt, L., Johnson, L., Clardy, D., Castillo-Rogez, J., Frick, A., and Jones, L. "Near-Earth Asteroid Scout," AIAA SPACE 2014 Conference and Exposition. American Institute of Aeronautics and Astronautics, San Diego, CA, 2014.

6. McInnes, C. R. Solar Sailing: Technology, Dynamics and Mission Applications. Berlin: Springer-Praxis Books in Astronautical Engineering, Springer-Verlag, 1999.

7. Baoyin, H., and McInnes, C. "Solar Sail Halo Orbits at the Sun-Earth Artificial L1-point," Celestial Mechanics and Dynamical Astronomy Vol. 94, 2006, pp. 155-171. doi: 10.1007/s10569-005-4626-3

8. Heiligers, J., and McInnes, C. "Novel Solar Sail Mission Concepts for Space Weather Forecasting," 24th AAS/AIAA Space Flight Mechanics Meeting. Santa Fe, NM, 2014.

9. Waters, T. J., and McInnes, C. R. "Periodic Orbits Above the Ecliptic in the Solar-Sail Restricted Three-Body Problem," Journal of Guidance, Control, and Dynamics Vol. 30, No. 3, 2007, pp. 687-693. doi: $10.2514 / 1.26232$

10. McInnes, C. R., McDonald, A. J., Simmons, J. F. L., and MacDonald, E. W. "Solar Sail Parking in Restricted Three-Body Systems," Journal of Guidance, Control, and Dynamics Vol. 17, No. 2, 1994, pp. 399-406. doi: $10.2514 / 3.21211$

11. McInnes, C. R. "Solar Sail Trajectories at the Lunar L2 Lagrange Point," Journal of Spacecraft and Rockets Vol. 30, No. 6, 1993, pp. 782-784. doi: 10.2514/3.26393

12. Simo, J., and McInnes, C. R. "Solar Sail Orbits at the Earth-Moon Libration Points," Communications in Nonlinear Science and Numerical Simulation Vol. 14, No. 12, 2009, pp. 4191-4196. doi: 10.1016/j.cnsns.2009.03.032 
13. Wawrzyniak, G. G., and Howell, K. C. "Generating Solar Sail Trajectories in the Earth-Moon System Using Augmented Finite-Difference Methods (Article ID 476197)," International Journal of Aerospace Engineering, 2011. doi: 10.1155/2011/476197

14. Heiligers, J., Diedrich, B., Derbes, B., and McInnes, C. R. "Sunjammer: Preliminary End-to-End Mission Design," 2014 AIAA/AAS Astrodynamics Specialist Conference. San Diego, CA, USA, 2014.

15. Howell, K. C. "Three-Dimensional, Periodic, 'Halo' Orbits," Celestial Mechanics and Dynamical Astronomy Vol. 32, 1983, pp. 53-71. doi: 10.1007/BF01358403

16. Wie, B. "Solar Sail Attitude Control and Dynamics, Part 1," Journal of Guidance, Control, and Dynamics Vol. 27, No. 4, 2004, pp. 526-535. doi: 10.2514/1.11134 